\title{
Computational Approaches to Connecting Levels of Analysis in Networked Learning Communities
}

\author{
H. Ulrich Hoppe \\ University of Duisburg-Essen \\ (Germany) \\ +492033793553 \\ hoppe@collide.info
}

\author{
Daniel D. Suthers \\ University of Hawai'i at Manoa \\ Honolulu (USA) \\ +1 808 956-3890 \\ suthers@hawaii.edu
}

\begin{abstract}
The focus of this workshop is on the potential benefits and challenges of using specific computational methods to analyze interactions in networked learning environments, particularly with respect to integrating multiple analytic approaches towards understanding learning at multiple levels of agency, from individual to collective. The workshop is designed for researchers interested in analytical studies of collaborative and networked learning in socio-technical networks, using data-intensive computational methods of analysis (including social-network analysis, log-file analysis, information extraction and data mining). The workshop may also be of interest to pedagogical professionals and educational decision makers who want to evaluate the potential of learning analytics techniques to better inform their decisions regarding learning in technology-rich environments.
\end{abstract}

\section{Keywords}

Computational interaction analysis, levels of analysis, networked learning, CSCL

\section{BACKGROUND}

This workshop continues the themes of "connecting levels" and "multivocality" that have characterized two series of workshops at several conferences (ARV, CSCL, ICLS, LAK). We have taken a multi-disciplinary perspective on learning as involving the agency of individuals, groups and communities, and sought to understand learning across these levels by integrating multiple methods and granularities of analysis (cf. [7, 8]).

The workshop for LAK 2014 focuses on specific computational methods and their potential for analyzing interactions in networked learning communities from different perspectives. Social network analysis (SNA, cf. [10]) is an approach that has been used in studying networked learning in the past (e.g., $[1,2$, $3]$ ), and it is still of interest. However, we are particularly interested in new methods and new combinations of different analysis techniques and computational approaches. Martinez et al. [4] provide an example of such a mixed method approach, coordinating SNA with other qualitative and quantitative analysis methods in a study of participatory aspects of learning in CSCL contexts. Further work in automating such approaches and exploring the complementarities of different data sources and analytic approaches is needed.
SNA has been criticized for eliminating time from the results of an analysis in that it aggregates data over certain time intervals without being able to show time-dependent patterns. This can be partially overcome by using time series of networks. Yet, Zeini et al. have shown that also the choice of measurement intervals has systematic effects on the resulting networks [11]. On the other hand, there are analytic methods, such as process mining, that are particularly geared to extracting procedural patterns from interaction data [5]. Another group of methods deals with extracting content information from (textual) artifacts, using both "shallow" and "deep" linguistic techniques. At this point, we do not expect any one of these methods to be sufficient alone or to succeed on a purely technical level. We would rather favor a triangulation approach in which several methods are applied to the same data sources and are interpreted in conjunction with each other and theoretical considerations.

Although the workshop is focused on (new) computational methods or new applications of such methods, we are also interested in discussing computational approaches in a conceptual and/or theoretical context. In this perspective, Suthers et al. [6,9] have developed a rich contextual framework to interpret collaborative interactions, introducing certain steps of interpretation and addressing different levels of granularity using concepts such as "contingencies" and "uptake" (as relations between actions or contributions). It is of particular interest to further automate the application of such interpretation schemes.

Given these premises, we have invited contributions guided by the following questions:

- How to detect emergent phenomena and patterns in traces of collective/collaborative learning activities by using a plurality of computational methods? How do we interconnect these methods?

- What practical techniques such as different types of triangulation or visualization can help to connect different levels and approaches of analysis?

- How can we integrate SNA with content analysis methods (including LDA, LSA, Network Text Analysis) and with the detection of interaction patterns?

- How can conceptually/theoretically grounded interpretation schemes for collaborative activities be adequately operationalized and automated?

- What are the prospects of technical integration of analysis tools through a kind of "open analysis workbench" (open architecture, GUI metaphors). 


\section{WORKSHOP CONTRIBUTIONS}

The workshop includes a mixture of presentations, interactive demos, and group discussions. The following contributions will be presented in the workshop:

Daniel Suthers and Nathan Dwyer: Multilevel Analysis of Uptake, Sessions, and Key Actors in a Socio-Technical Network

Agathe Merceron: Connecting Analysis of Speech Acts and Performance Analysis - An Initial Study

Hiroaki Ogata, Songran Liu and Kousuke Mouri: Ubiquitous Learning Analytics Using Learning Logs

Hiroaki Ogata: Supporting Science Communication in a Museum using Ubiquitous Learning Logs

Tilman Göhnert, Sabrina Ziebarth, Per Verheyen, and H. Ulrich Hoppe: Integration of a Flexible Analytics Workbench with a Learning Platform for Medical Specialty Training

H. Ulrich Hoppe, Tilman Göhnert, Laura Steinert, and Christopher Charles: A Web-based Tool for Communication Flow Analysis of Online Chats

Wanli Xing and Sean Goggins: Automated CSCL Group Assessment: Activity Theory based Clustering Method

Cindy Hmelo-Silver, Carolyn Rosé, and Jeff Levy: Fostering a Learning Community in MOOCs

\section{WORKSHOP FACILITATORS}

\section{Ulrich Hoppe}

H. Ulrich Hoppe holds a full professorship for "Cooperative and Learning Support Systems" in the Department of Computer Science and Applied Cognitive Science at the University of Duisburg-Essen, Germany. With his research group COLLIDE, Ulrich Hoppe has been engaged in several European projects in the area of advanced computational technologies in education since 1998. Ulrich Hoppe has been program chair of AIED and CSCL 2003, ICCE 2007 and CRIWG 2012. His current research interests include: interactive and collaborative media for learning and knowledge construction; analysis, modelling, and intelligent support of interactive and collaborative learning processes; social network analysis and community support.

\section{Dan Suthers}

Daniel D. Suthers is Professor in the Department of Information and Computer Sciences at the University of Hawai i at Manoa, where he directs the Laboratory for Interactive Learning Technologies. Dr. Suthers' research is generally concerned with cognitive, social and computational perspectives on designing and evaluating software for learning, collaboration, and community. Dr. Suthers initiated and chaired a series of five workshops on Productive Multivocality in Analysis of Collaborative Learning, involving dozens of researchers in a long term collaboration leading to a book in press. Subsequently he led workshops on Connecting Levels of Analysis at the CSCL 2011 and 2013 and LAK 2012 conferences. He has also served as program chair for LAK, ICCE, and two CSCL conferences, and has had steering committee roles (e.g., workshop chair, interactive events chair) for numerous other conferences.

\section{Committee}

- $\quad$ Tilman Göhnert, University of Duisburg-Essen, Germany

- $\quad$ Sean Goggins, Drexel University, USA

- Vanda Luego, Université Joseph Fourier, France

- Agathe Merceron, Beuth University of Applied Sciences, Germany

- Hiroaki Ogata, Kyushu University, Japan

- John Stamper, Carnegie Mellon University, USA

- $\quad$ Chris Teplovs, Problemshift Inc., Canada

\section{REFERENCES}

[1] M. de Laat, V. Lally, L. Lipponen and R.-J. Simons, Investigating patterns of interaction in networked learning and computer-supported collaborative learning: A role for Social Network Analysis, International Journal of Computer Supported Collaborative Learning, 2 (2007), pp. 87-103.

[2] A. Harrer, N. Malzahn, S. Zeini, and H.U. Hoppe, Combining Social Network Analysis with semantic relations to support the evolution of a scientific community, in C. Chinn, G. Erkens and S. Puntambekar, eds., The Computer Supported Collaborative Learning (CSCL) Conference 2007, International Society of the Learning Sciences, New Brunswick, 2007, pp. 267-276.

[3] C. Haythornthwaite, Social network methods and measures for examining e-learning, E-learning seminar, University of Southampton, 2005.

[4] A. Martinez, Y. Dimitriadis, E. Gomez-Sanchez, B. RubiaAvi, I. Jorrin-Abellan and J. A. Marcos, Studying participation networks in collaboration using mixed methods, International Journal of Computer-Supported Collaborative Learning, 1 (2006), pp. 383-408.

[5] P. Reimann, Time is precious: Variable- and event-centred approaches to process analysis in CSCL research, Computer Supported Collaborative Learning, 4 (2009), pp. 239-257.

[6] D. D. Suthers, N. Dwyer, R. Medina and R. Vatrapu, A framework for conceptualizing, representing, and analyzing distributed interaction, International Journal of Computer Supported Collaborative Learning, 5 (2010), pp. 5-42.

[7] D. D. Suthers, H. U. Hoppe, M. De Laat, and S. Buckingham Shum. LAK, Connecting levels and methods of analysis in networked learning communities. Proceedings of LAK 2012. ACM, (2012), pp. 11-13.

[8] D. D. Suthers, K. Lund, C. Rosé, G. Dyke, C. Teplovs and N. Law. Productive Multivocality in the Analysis of Group Interactions. New York: Springer, 2013.

[9] D. D. Suthers and D. Rosen, A unified framework for multilevel analysis of distributed learning Proceedings of the First International Conference on Learning Analytics \& Knowledge, Banff, Alberta, February 27-March 1, 2011, 2011.

[10] S. Wasserman and K. Faust, Social Network Analysis: Methods and Applications, Cambridge University Press, New York, 1994.

[11] S. Zeini, T. Göhnert, L. Krempel and Hoppe H. U. (2012). The impact of measurement time on subgroup detection in online communities. The 2012 IEEE/ACM International Conference on Advances in Social Networks Analysis and Mining (ASONAM 2012). 